\title{
Hémoparasites Chez Les Ruminants Domestiques Dans Les Départements De Korhogo Et Sinématiali En Côte d'Ivoire
}

\author{
Nawolo Yéo, \\ Biégo Guillaume Gragnon, \\ Laboratoire Régional de Korhogo, Laboratoire National d'Appui au \\ Développement Agricole, Côte d'Ivoire \\ Yahaya Karamoko, \\ Unité de Formation et de Recherche des Sciences de la Nature, \\ Université Nangui Abrogoua, Abidjan, Côte d'Ivoire
}

Doi:10.19044/esj.2020.v16n15p183 URL:http://dx.doi.org/10.19044/esj.2020.v16n15p183

\section{Resume}

L'étude réalisée de janvier à décembre 2019 dans le Nord de la Côte d'Ivoire a pour but de déterminer la prévalence des hémoparasites chez les bovins et ovins d'élevage. Des frottis sanguins ont été réalisés à partir du sang périphérique de l'oreille de 68 bovins et 64 ovins provenant des localités de Korhogo, Nafoun, Nagougaha, Mongaha et Lokoli. Les résultats obtenus de l'étude proviennent du registre de laboratoire collectés sur l'année 2019. L'observation microscopique des frottis sanguins a permis d'identifier trois genres d'hémoparasites: Anaplasma, Babesia et Theileria. Theileria est absent chez les ovins. Concernant l'espèce d'hémoparasite, les ovins $(71,88 \%)$ et les bovins (70,59\%) sont plus infectés par Anaplasma marginale avec une différence significative ( $\mathrm{p}$-value $=0,0001$ ). Par ailleurs, les bovins de plus d'un an sont plus infectés par Anaplasma marginale. La prévalence d'infection est de $32,35 \%$ pour ceux d'un an à 3 ans et $29,41 \%$ pour ceux de plus de 3ans d'âge. Les femelles $(54,41 \%)$ sont les plus infectées. Les ovins de plus de 3 mois d'âge sont plus infectés. En outre, les femelles $(45,31 \%)$ sont les plus infectées avec une différence significative $(\mathrm{p}$-value $=0,0261)$ pour Anaplasma marginale. Les bovins de la localité de Korhogo sont plus infectés par Anaplasma marginale (36,76\%) suivis des bovins de Nagougaha $(20,59 \%)$ avec une différence significative ( $\mathrm{p}$-value $=0,0077)$. Par ailleurs, Anaplasma centrale (18,75\%) infecte plus les ovins de la localité de Lokoli suivi de Korhogo $(4,69 \%)$ avec une différence significative ( $\mathrm{p}$-value $=$ 0,00037).

Mots clés : Hémoparasite, Prévalence, Localité, Département, Côte d'Ivoire 


\title{
Hemoparasites in Domestic Ruminants in the Korhogo and Sinematiali Departments of Côte d'Ivoire
}

\author{
Nawolo Yeo, \\ Biégo Guillaume Gragnon, \\ Laboratoire Régional de Korhogo, Laboratoire National d'Appui au \\ Développement Agricole, Côte d'Ivoire \\ Yahaya Karamoko, \\ Unité de Formation et de Recherche des Sciences de la Nature, \\ Université Nangui Abrogoua, Abidjan, Côte d'Ivoire
}

\begin{abstract}
The study carried out from January to December 2019 in the North of Côte d'Ivoire aims to determine the prevalence of hemoparasites in cattle and sheep breeding. Blood smears were taken from the peripheral ears of 68 cattle and 64 sheep from the localities of Korhogo, Nafoun, Nagougaha, Mongaha and Lokoli. The results obtained from the study come from the laboratory register collected in 2019. Microscopic examination of blood smears has identified three types of hemoparasites: Anaplasma, Babesia and Theileria. Theileria is absent in sheep. At the level of hemoparasite species, sheep (71.88\%) and cattle (70.59\%) are more infected by Anaplasma marginale with a significant difference $(\mathrm{p}$-value $=0.0001)$. In addition, cattle over one year are more infected with Anaplasma marginale. The prevalence of infection is $32.35 \%$ for one year to 3 years and $29.41 \%$ for over 3 years of age. Females $(54.41 \%)$ are the most infected. Sheep over 3 months of age are more infected. Females $(45.31 \%)$ are the most infected with a significant difference (p-value $=0.0261$ ) for Anaplasma marginale. Cattle of Korhogo are more infected with Anaplasma marginale (36.76\%) followed by Nagougaha $(20.59 \%)$ with a significant difference ( $\mathrm{p}$-value $=0.0077$ ). In addition, Anaplasma centrale (18.75\%) more infects sheep in the locality of Lokoli followed by Korhogo $(4.69 \%)$ with a significant difference $(p$-value $=0.00037)$.
\end{abstract}

Keywords: Hemoparasite, Prevalence, Locality, Department, Côte d'Ivoire 


\section{Introduction}

En Côte d'Ivoire, où le développement économique est fondé sur l'agriculture, l'élevage est resté une activité secondaire. Les productions animales issues de ces élevages sont faiblement exploitées. Les déficits en viande et produits laitiers sont respectivement 55,4\% et 87,4\% (Yabile, 2011). Cependant, de nombreuses contraintes tant alimentaires que sanitaires limitent le développement des productions animales. Au nombre de ces contraintes, les parasites sanguins notamment ceux du genre Anaplasma, Babesia et Theileria (Farougou et al., 2012) occupent une place de choix. Ces hémoparasites sont responsables de nombreuses mortalités dans les élevages. L'impact de ces hémoparasites entraine les avortements, les chutes de poids et la baisse de la production laitière (Farougou et al., 2007). Selon l'Organisation Mondiale de la Santé Animale (ex Office International des Epizooties) (OIE) en 2008, la Babésiose bovine est mondialement la plus importante des parasitoses. Son effet accidentel zoonotique a été déjà montré chez l'homme en France et, peut se présenter comme une zoonose émergente. Cette maladie entrave l'amélioration des races rustiques locales par croisement avec des reproducteurs de races améliorées et, est en croissance dans le monde (Lachance et al., 2012) avec cependant, une prévalence plus importante en zone tropicale (Stachurski et al., 2004 ; Oguz, 2008). Babesia chez les ruminants domestiques, comprend deux espèces : Babesia bovis et Babesia bigemina. Ces parasites peuvent être communs aux bovins et aux ovins d'élevage (Adamu et Balarabe, 2012). Les tiques, considérées comme le deuxième groupe de vecteurs de maladies humaines après les moustiques (Goodman et al., 2005), constituent le vecteur principal des parasites du genre Anaplasma, Babesia et Theileria. La persistance des tiques sur les bovins et les ovins d'élevage et l'absence de synchronisation des traitements de ces espèces animales contre les hémoparasites par les éleveurs du Nord de la Côte d'Ivoire seraient à l'origine de la résistance des espèces animales face à l'emploi des acaricides et des antiparasitaires sanguins. Au vu de ce qui précède, il est donc nécessaire de rechercher les hémoparasites chez les bovins et les ovins afin d'adapter un programme de lutte contre ces affections parasitaires. Les données d'analyse de routine du Laboratoire Régional de Korhogo (LRK) du Laboratoire National d'Appui au Développement Agricole (LANADA) viennent renforcer celles existantes sur les hémoparasites chez les bovins et les ovins d'élevage et apporter un appui scientifique aux éleveurs. L'objectif de l'étude est de déterminer la prévalence des hémoparasites chez les ruminants domestiques dans les départements de Korhogo et Sinématiali en Côte d'Ivoire. 


\section{Materiel Et Methodes}

\section{Zone D'étude}

Le Nord de la Côte d'Ivoire est composé de plus de $80 \%$ de formations savanicoles qui sont la savane boisée, la savane arborée, la savane arbustive et la savane herbeuse. Le climat est de type soudanien avec une saison unique des pluies qui dure six mois et demie (mi-avril à octobre), avec un pic de juillet à octobre ; une saison sèche dont l'influence maximale se situe de novembre à fin mars. Les données de l'étude proviennent d'enquêtes dans les départements de Korhogo (Korhogo, Nafoun, Nagougaha et Mongaha) et de Sinématiali (Lokoli) et de diagnostic de routine de laboratoire réalisés au Laboratoire Régional de Korhogo du Laboratoire National d'Appui au Développement Agricole dans le Nord de la Côte d'Ivoire (Figure 1). Le choix de la zone d'étude a porté sur les départements de Korhogo et de Sinématiali du fait de sa forte production de bétail. En effet, selon Tanguy (2008), les seuls départements de Korhogo et de Ferkessédougou constituent près de la moitié du cheptel national de la Côte d'Ivoire.

\section{Enquête dans les localités visitées Période d'étude}

L'étude s'est déroulée de janvier à décembre 2019 dans les élevages de ruminants domestiques.

\section{Choix des animaux}

Le Nord de la Côte d'Ivoire est reconnu comme une zone de forte production des ruminants domestiques (bovins et ovins) du fait de la densité en herbe constituant la base de l'alimentation des bovins et des ovins. Ces animaux ont été choisis au hasard sans distinction de sexe et d'âge. Les bovins qui ont fait l'objet d'étude ont été essentiellement des bovins métisses croisés Taurin x Zébu. Quant aux ovins, ils ont appartenu tous à la race djallonké, majoritaire dans la zone d'étude.

\section{Prélèvement de sang et recherche d'hémoparasites}

Un examen hématologique a été réalisé chez 132 animaux dont 68 bovins et 64 ovins. Le sang périphérique a été prélevé au niveau de la base de l'oreille de l'animal à l'aide d'un tube micro capillaire à hématocrite. Ce sang a servi à la confection de 132 frottis sanguins.

Chez les bovins, 29 frottis sanguins ont été collectés dans la localité de Korhogo, 13 frottis à Nafoun, 18 frottis à Nagougaha et 8 frottis à Mongaha. Tandis que chez les ovins, 23 frottis sanguins ont porté sur la localité de Korhogo, 23 frottis à Nafoun et 18 frottis à Lokoli. Le stade physiologique des espèces animales a été pris en compte dans l'étude (Tableau 1 et 2). Ces frottis sanguins séchés à l'abri de la poussière, du soleil et des mouches ont été 
ensuite fixés au Laboratoire Régional de Korhogo au méthanol 95\% durant 5 minutes puis séchés environ une heure et colorés au Giemsa dilué $1 / 10$ pendant 30 minutes. Après la coloration, les frottis sanguins ont été rincés sous un filet d'eau et séchés pendant au moins une heure avant d'être examinés au microscope optique à l'objectif 100. Le tableau 1 et le tableau 2 présentent respectivement l'effectif des bovins et des ovins en fonction de l'âge et du sexe des animaux. Les résultats obtenus de l'étude proviennent des résultats du registre de laboratoire collectés sur l'année 2019.

Tableau 1: Effectifs des bovins infectés en fonction du type d'infection

\begin{tabular}{|c|c|c|}
\hline \multirow{3}{*}{ Paramètres } & Stade Physiologique & $\begin{array}{c}\text { Nombre de } \\
\text { prélèvements }\end{array}$ \\
\hline \multirow{4}{*}{ Age } & $\begin{array}{c}\text { Veau/vêle } \\
\mathbf{0 - 1 a n}\end{array}$ & 15 \\
\cline { 2 - 3 } & Taurillon/génisse 1 - 3 ans & 29 \\
\cline { 2 - 3 } & Taureau/vache plus de 3 ans & 24 \\
\cline { 2 - 3 } & Total & $\mathbf{6 8}$ \\
\hline \multirow{3}{*}{ Sexe } & $\mathrm{F}$ & 51 \\
\cline { 2 - 3 } & M & 17 \\
\cline { 2 - 3 } & Total & $\mathbf{6 8}$ \\
\hline
\end{tabular}

Tableau 2: Effectifs des ovins infectés en fonction du type d'infection

\begin{tabular}{|c|c|c|}
\hline \multirow{3}{*}{ Paramètres } & Stade Physiologique & $\begin{array}{c}\text { Nombre de } \\
\text { prélèvements }\end{array}$ \\
\hline \multirow{5}{*}{ Age } & $\begin{array}{c}\text { Agneau/agnelle } \\
\text { 0- 3mois }\end{array}$ & 5 \\
\cline { 2 - 3 } & $\begin{array}{c}\text { Antenais/antenaise 3mois - } \\
\text { 1an }\end{array}$ & 31 \\
\cline { 2 - 3 } & $\begin{array}{c}\text { Bélier/brebis } \\
\text { plus d'un an }\end{array}$ & 28 \\
\cline { 2 - 3 } & Total & $\mathbf{6 4}$ \\
\hline \multirow{3}{*}{ Sexe } & F & 40 \\
\cline { 2 - 3 } & M & 24 \\
\cline { 2 - 3 } & Total & $\mathbf{6 4}$ \\
\hline
\end{tabular}

\section{Analyse des données et traitement statistique}

La fréquence des espèces animales ayant fait l'objet d'analyse de routine a été calculée comme suit :

$$
F(\%)=\frac{\text { Effectif d'une espèce animale donnée }}{\text { Effectif total des animaux }} \times 100
$$

Quant à la prévalence des hémoparasites par espèce animale et par localité, elle a été calculée selon la formule suivante : 


$$
P(\%)=\frac{\text { Nombre de sujets positifs à un type d'infection }}{\text { Nombre total de sujets échantillonnés }} \times 100
$$

Les comparaisons des fréquences et des prévalences des hémoparasites par espèce animale et par localité ont été réalisées en utilisant le test de Khi deux $\left(\chi^{2}\right)$. La différence entre ces fréquences et ces prévalences a été considérée significative au seuil de $5 \%$.

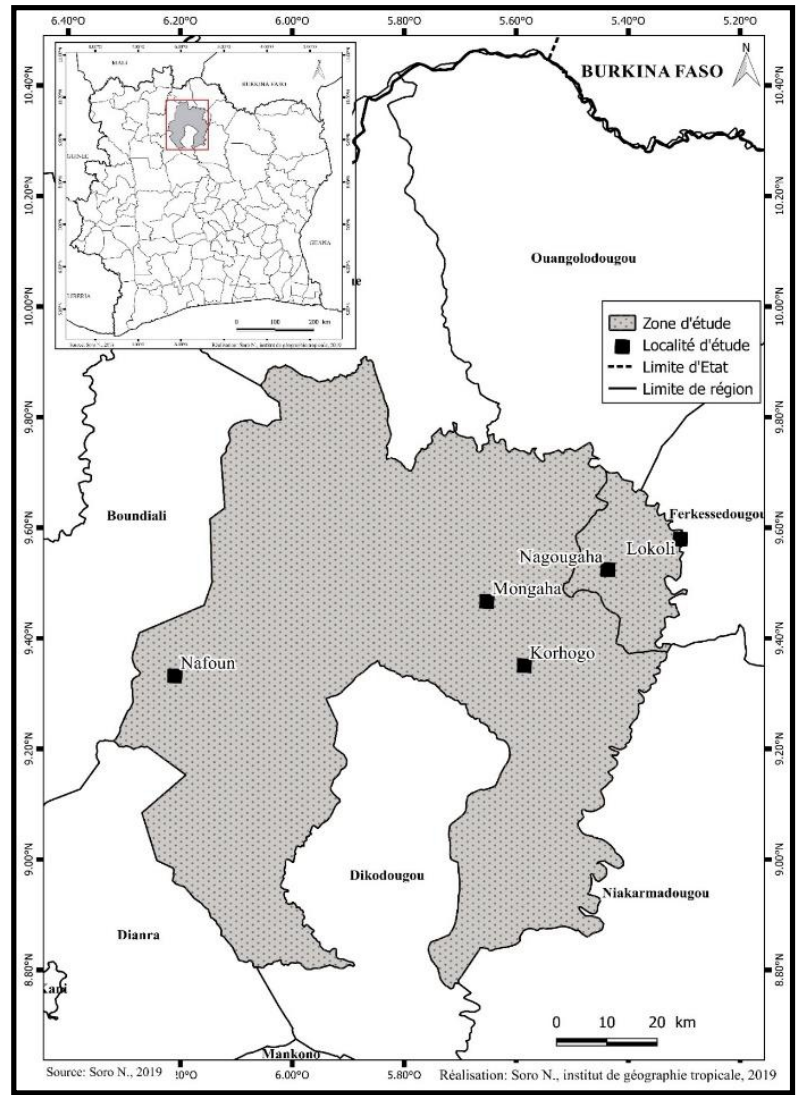

Figure 1 : Situation géographique des localités d'étude dans la Région du Poro en Côte d'Ivoire

\section{Resultats}

\section{Fréquence des animaux ayant fait l'objet d'analyse de routine}

Sur 132 animaux, les bovins et les ovins ayant fait l'objet d'analyse de routine sont en nombre presqu'identique durant l'année 2019. L'effectif des bovins est de 68 contre 64 ovins. Les fréquences respectives pour ces espèces animales sont $51,52 \%$ et $48,48 \%$. La différence statistique des fréquences entre ces espèces animales n'est pas significative ( $p$-value $=0,7611)$ (Tableau $3)$. 
Tableau 3 : Fréquence des animaux ayant fait l'objet d'analyse de routine

\begin{tabular}{|c|c|c|c|c|}
\hline $\begin{array}{c}\text { Espèce } \\
\text { animale }\end{array}$ & $\begin{array}{c}\text { Effectif } \\
\text { animaux }\end{array}$ & $\begin{array}{c}\text { Fréquence } \\
(\%)\end{array}$ & $\mathrm{Khi}^{2}$ & $\mathrm{P}$ - value \\
\hline Bovins & 68 & 51,52 & \multirow{2}{*}{0,0924} & 0,7611 \\
\hline Ovins & 64 & 48,48 & - & - \\
\hline Total & 132 & 100 & & \\
\hline
\end{tabular}

\section{Prévalence globale des infections parasitaires sanguines dans la zone d'étude}

Globalement, trois (3) genres de parasites sanguins infectent les animaux de la zone d'étude. Il s'agit des parasites du genre Anaplasma, Babesia et Theileria. Cependant, le genre Anaplasma s'est révélé plus infectant avec une prévalence de 70,29\% suivies respectivement du genre Babesia $(18,84 \%)$ et Theileria $(7,97 \%)$. Par ailleurs, la différence statistique de prévalence s'est révélée significative $(\mathrm{p}$-value $=0,0001)$ (Figure 2$)$.

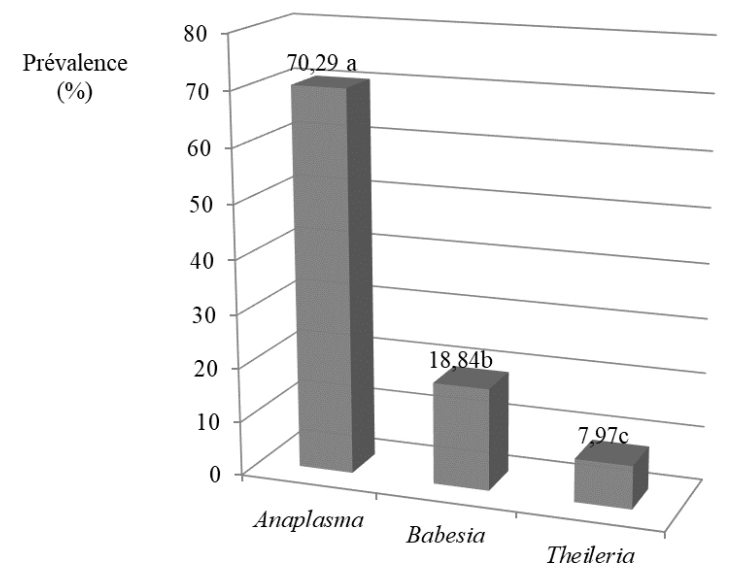

Type d'hémoparasites

La différence de lettres ( $a, b$ et $c)$ indiquées, montre qu'il y a une différence significative entre les prévalences des types d'hémoparasites.

Figure 2 : Prévalence globale des infections parasitaires sanguines dans la zone d'étude

\section{Prévalence des infections parasitaires sanguines des animaux en fonction du genre du parasite sanguin}

Selon les espèces animales, les bovins sont infectés par les trois genres de parasites sanguins avec une prévalence élevée pour le genre Anaplasma (70,59\%) suivis du genre Babesia $(29,41 \%)$ et le genre Theileria $(16,18 \%)$. Il est de même pour l'espèces ovine où le parasite sanguin du genre Theileria est absent. Les prévalences d'infection de ces parasites sanguins diffèrent significativement $(\mathrm{p}$-value $=0,0001)$ au niveau de l'espèce bovine et ovine (Figure 3 et 4 ). 

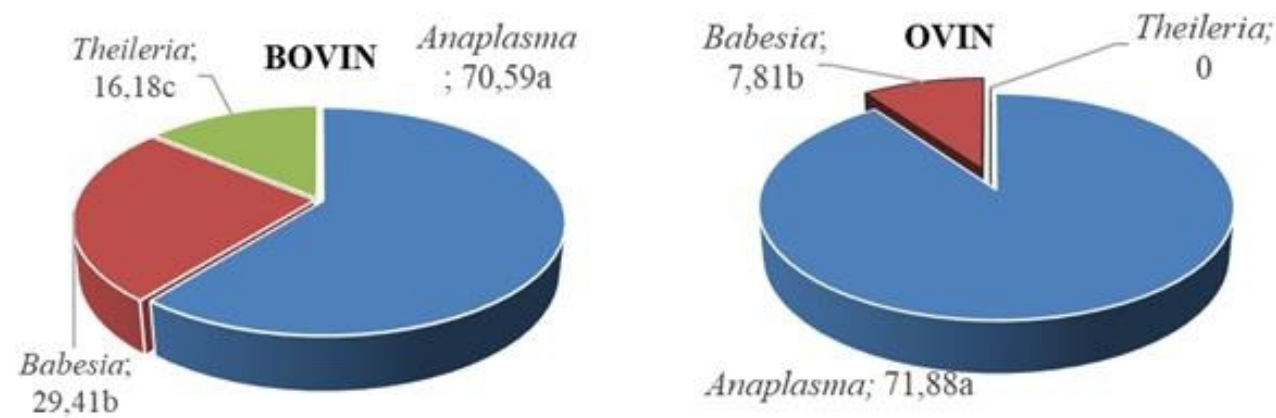

La différence de lettres ( $a, b$ et $c$ ) indiquées, montre qu'il y a une différence significative entre les prévalences des genres d'hémoparasites, selon l'espèce animale.

Figure 3 : Prévalence des infections parasitaires sanguines chez les bovins en fonction du genre du parasite sanguin

Figure 4 : Prévalence des infections parasitaires sanguines chez les ovins en fonction du genre du parasite sanguin

\section{Prévalence des espèces d'hémoparasites en fonction de l'espèce animale}

Concernant les espèces de parasites sanguins, Anaplasma marginale est de loin le parasite sanguin qui infecte le plus les bovins suivis de Babesia bovis. Le taux d'infection des bovins par ces parasites est respectivement de $70,59 \%$ et $29,41 \%$. Les hémoparasites notamment, Anaplasma centrale, Theileria spp et Babesia bigemina infectent les bovins à des prévalences moindre. La différence de prévalence des infections par ces hémoparasites s'est révélée significative ( $\mathrm{p}$-value $=0,0001$ ) chez l'espèce bovine (Figure 5). En outre, chez les ovins, Anaplasma marginale et Anaplasma centrale infectent plus les ovins de la zone d'étude. Les prévalences d'infection des ovins pour ces hémoparasites sont respectivement de $71,88 \%$ et $26,56 \%$. Par ailleurs, Babesia ovis et Babesia bigemina infectent faiblement les ovins. Contrairement aux infections bovines, Theileria spp est absent chez les ovins. 

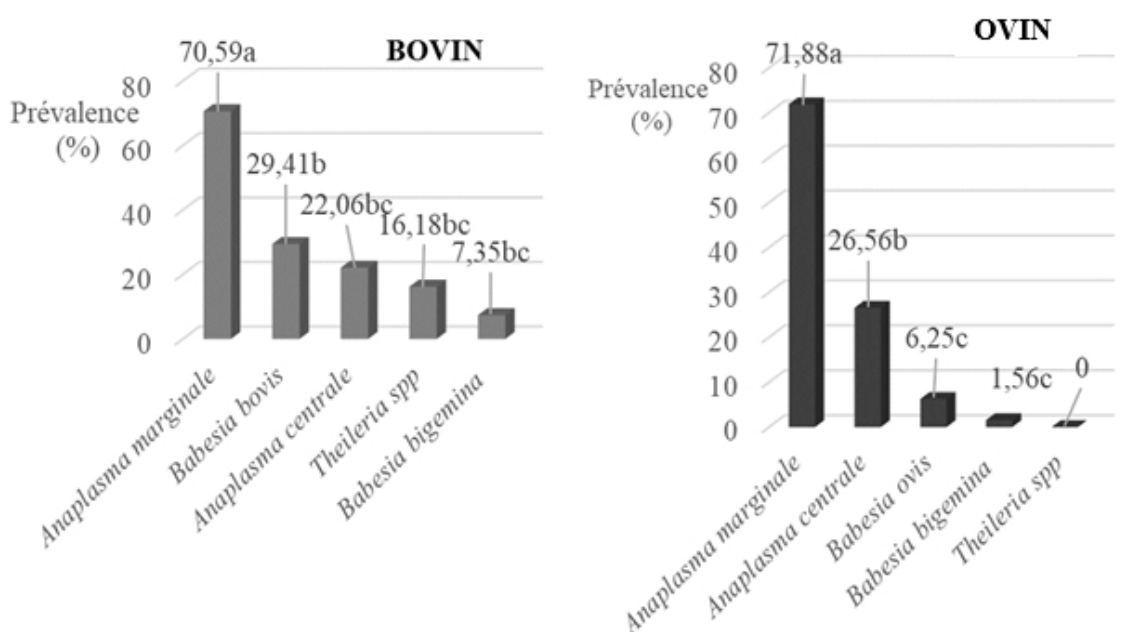

La différence de prévalence des infections ovines pour ces parasites est significative $(p=0,0001)$ dans la zone d'étude (Figure 6).

Figure 5 : Prévalence des infections bovines selon le type d'hémoparasites

Figure 6 : Prévalence des infections ovines selon le type d'hémoparasites

\section{Prévalence des espèces d'hémoparasites en fonction du stade physiologique des bovins}

Le tableau 4 montre la prévalence des espèces d'hémoparasites en fonction de l'âge et du sexe des bovins. Au niveau de l'âge des bovins, les taurillons et les génisses de 1 à 3 ans ainsi que les taureaux et les vaches de plus de 3 ans d'âge sont les plus infectés par Anaplasma marginale. Le taux d'infection de ces classes d'âge est respectivement $32,35 \%$ et $29,41 \%$. Par ailleurs, Anaplasma marginale infecte faiblement les veaux et les vêles de moins d'un an à une prévalence de $8,82 \%$. Statistiquement, il y a une différence significative de prévalence $(\mathrm{p}$-value $=0,00013)$ pour ce parasite sanguin au niveau des classes d'âge des bovins.

Concernant le sexe des bovins, les femelles sont plus infectées par les espèces d'hémoparasites du genre Anaplasma. Le taux d'infection des femelles est de 54,41\% contre 16,18\% chez les mâles pour Anaplasma marginale. La différence statistique de prévalence pour ce parasite s'est révélée significative à $\mathrm{p}$-value $=0,0001$ au niveau du paramètre sexe des bovins. 
Tableau 4 : Prévalence des espèces d'hémoparasites en fonction du stade physiologique des bovins

\begin{tabular}{|c|c|c|c|c|c|c|}
\hline \multirow[b]{2}{*}{$\begin{array}{c}\text { Paramètre } \\
\mathrm{S}\end{array}$} & \multirow[b]{2}{*}{$\begin{array}{c}\text { Stade } \\
\text { Physiologique }\end{array}$} & \multicolumn{5}{|c|}{ Hémoparasites } \\
\hline & & $\begin{array}{c}\text { Anaplasm } \\
a \\
\text { marginale }\end{array}$ & $\begin{array}{l}\text { Anaplasm } \\
\text { a centrale }\end{array}$ & $\begin{array}{l}\text { Babesi } \\
\text { a bovis }\end{array}$ & $\begin{array}{c}\text { Babesia } \\
\text { bigemin } \\
\quad a\end{array}$ & $\begin{array}{c}\text { Theileri } \\
a \text { spp }\end{array}$ \\
\hline \multirow{3}{*}{ Age } & $\begin{array}{c}\text { Veau/ vêle } \\
\text { 0-1an }\end{array}$ & $\begin{array}{c}8,82^{\mathrm{a}} \\
(6 / 68)\end{array}$ & $\begin{array}{c}4,41^{\mathrm{a}} \\
(3 / 68) \\
\end{array}$ & $\begin{array}{l}7,35^{\mathrm{a}} \\
(5 / 68) \\
\end{array}$ & $\begin{array}{l}2,94^{\mathrm{a}} \\
(2 / 68) \\
\end{array}$ & $\begin{array}{c}0 \\
(0 / 68) \\
\end{array}$ \\
\hline & $\begin{array}{c}\text { Taurillon/géniss } \\
\text { e } \mathbf{1} \text { - } \mathbf{3} \text { ans }\end{array}$ & $\begin{array}{l}32,35^{\mathrm{b}} \\
(22 / 68)\end{array}$ & $\begin{array}{l}13,24^{\mathrm{b}} \\
(9 / 68)\end{array}$ & $\begin{array}{l}10,29^{a} \\
(7 / 68)\end{array}$ & $\begin{array}{l}2,94^{\mathrm{a}} \\
(2 / 68)\end{array}$ & $\begin{array}{l}4,41^{\mathrm{a}} \\
(3 / 68)\end{array}$ \\
\hline & $\begin{array}{l}\text { Taureau/vache } \\
\text { plus de } 3 \text { ans }\end{array}$ & $\begin{array}{l}29,41^{b} \\
(20 / 68)\end{array}$ & $\begin{array}{l}4,41^{\mathrm{a}} \\
(3 / 68)\end{array}$ & $\begin{array}{l}11,76^{\mathrm{a}} \\
(8 / 68)\end{array}$ & $\begin{array}{c}1,47^{a} \\
(1 / 68)\end{array}$ & $\begin{array}{l}11,76^{a} \\
(8 / 68)\end{array}$ \\
\hline \multirow{2}{*}{ Sexe } & $\mathrm{F}$ & $\begin{array}{l}54,41^{\mathrm{a}} \\
(37 / 68)\end{array}$ & $\begin{array}{c}14,71^{\mathrm{a}} \\
(10 / 68)\end{array}$ & $\begin{array}{c}20,59^{a} \\
(14 / 68)\end{array}$ & $\begin{array}{l}4,41^{a} \\
(3 / 68)\end{array}$ & $\begin{array}{l}13,24^{a} \\
(9 / 68)\end{array}$ \\
\hline & $\mathrm{M}$ & $\begin{array}{l}16,18^{b} \\
(11 / 68)\end{array}$ & $\begin{array}{l}7,35^{\mathrm{a}} \\
(5 / 68) \\
\end{array}$ & $\begin{array}{c}8,82^{b} \\
(6 / 68)\end{array}$ & $\begin{array}{l}2,94^{a} \\
(2 / 68)\end{array}$ & $\begin{array}{l}2,94^{\mathrm{b}} \\
(2 / 68)\end{array}$ \\
\hline
\end{tabular}

*Significatif

La différence de lettres ( $\mathrm{a}, \mathrm{b}$ et $\mathrm{c}$ ) indiquées au niveau des colonnes, montre qu'il y a une différence significative entre les prévalences des espèces d'hémoparasites, en fonction de l'âge et du sexe du bovin.

\section{Prévalence des espèces d'hémoparasites en fonction du stade physiologique des ovins}

L'analyse du tableau 5 montre que les antenais, les antenaises, les béliers et les brebis sont les plus infectés par les hémoparasites de tous genres. Cependant, Anaplasma marginale est le parasite sanguin qui infecte le plus les ovins de tout âge. La forte infection des ovins par ce parasite est observée chez les adultes et les jeunes ovins avec une prévalence respective de 35,94\% et $31,25 \%$. Les agneaux et les agnelles sont infectés seulement par ce parasite sanguin à une prévalence faible de 4,69\% par rapport aux adultes et aux jeunes. La différence statistique est significative $(\mathrm{p}$-value $=0,0001$ ) pour ce parasite sanguin.

Par ailleurs, au niveau du sexe des ovins, comme chez les bovins, les femelles sont les plus infectées par les hémoparasites de tous genres. Les hémoparasites du genre Anaplasma infectent plus les femelles. Le taux d'infection des femelles est de $45,31 \%$ pour Anaplasma marginale et $20,31 \%$ pour Anaplasma centrale. Statistiquement, il y a une différence significative ( $\mathrm{p}$-value $=0,0261)$ pour Anaplasma marginale et $\mathrm{p}$-value $=0,0051$ pour Anaplasma centrale au niveau du paramètre sexe des ovins (Tableau 5) 
Tableau 5 : Prévalence des espèces d'hémoparasites en fonction du stade physiologique des ovins

\begin{tabular}{|l|l|l|l|l|l|}
\hline \multirow{5}{*}{ Paramètres } & \multicolumn{5}{|l|}{ Hémoparasites } \\
\cline { 3 - 7 } & Stade Physiologique & $\begin{array}{l}\text { Anaplasma } \\
\text { marginale }\end{array}$ & $\begin{array}{l}\text { Anaplasma } \\
\text { centrale }\end{array}$ & $\begin{array}{l}\text { Babesia } \\
\text { ovis }\end{array}$ & $\begin{array}{l}\text { Babesia } \\
\text { bigemina }\end{array}$ \\
\hline \multirow{5}{*}{ Age } & Agneau/agnelle & $4,69^{\mathrm{a}}$ & 0 & 0 & 0 \\
& $\mathbf{0 - 3 m o i s}$ & $(3 / 64)$ & $(0 / 64)$ & $(0 / 64)$ & $(0 / 64)$ \\
\cline { 2 - 7 } & Antenais/antenaise & $31,25^{\mathrm{b}}$ & $9,38^{\mathrm{a}}$ & $1,56^{\mathrm{a}}$ & 0 \\
& 3mois - 1an & $(20 / 64)$ & $(6 / 64)$ & $(1 / 64)$ & $(0 / 64)$ \\
\cline { 2 - 7 } & Belier/brebis & $35,94^{\mathrm{b}}$ & $17,19^{\mathrm{a}}$ & $4,69^{\mathrm{a}}$ & 1,56 \\
& plus d'un an & $(23 / 64)$ & $(11 / 64)$ & $(3 / 64)$ & $(1 / 64)$ \\
\hline \multirow{5}{*}{ Sexe } & & $45,31^{\mathrm{a}}$ & $20,31^{\mathrm{a}}$ & $4,69^{\mathrm{a}}$ & 1,56 \\
& $\mathrm{~F}$ & $(29 / 64)$ & $(13 / 64)$ & $(3 / 64)$ & $(1 / 64)$ \\
\cline { 2 - 7 } & & $26,56^{\mathrm{b}}$ & $6,25^{\mathrm{b}}$ & $1,56^{\mathrm{a}}$ & 0 \\
& $\mathrm{M}$ & $(17 / 64)$ & $(4 / 64)$ & $(1 / 64)$ & $(0 / 64)$ \\
\hline
\end{tabular}

*Significatif

La différence de lettres ( $\mathrm{a}, \mathrm{b}$ et $\mathrm{c}$ ) indiquées au niveau des colonnes, montre qu'il y a une différence significative entre les prévalences des espèces d'hémoparasites, en fonction de l'âge et du sexe de l'ovin.

\section{Prévalence des espèces d'hémoparasites bovins en fonction de la localité de prélèvement}

Au niveau des sites de prélèvement, les bovins de la localité de Korhogo sont plus infectés par les parasites sanguins du genre Anaplasma et Theileria. Les bovins de cette localité sont plus infectés par Anaplasma marginale (36,76\%) suivis de Babesia bovis et Theileria spp $(13,24 \%)$. Les bovins sont moins infectés par Anaplasma centrale (10,29\%). La différence statistique de prévalence est significative pour Anaplasma marginale ( $\mathrm{p}$-value $=0,0001)$ et Theileria $\mathrm{spp}(\mathrm{p}$-value $=0,0077)$. 


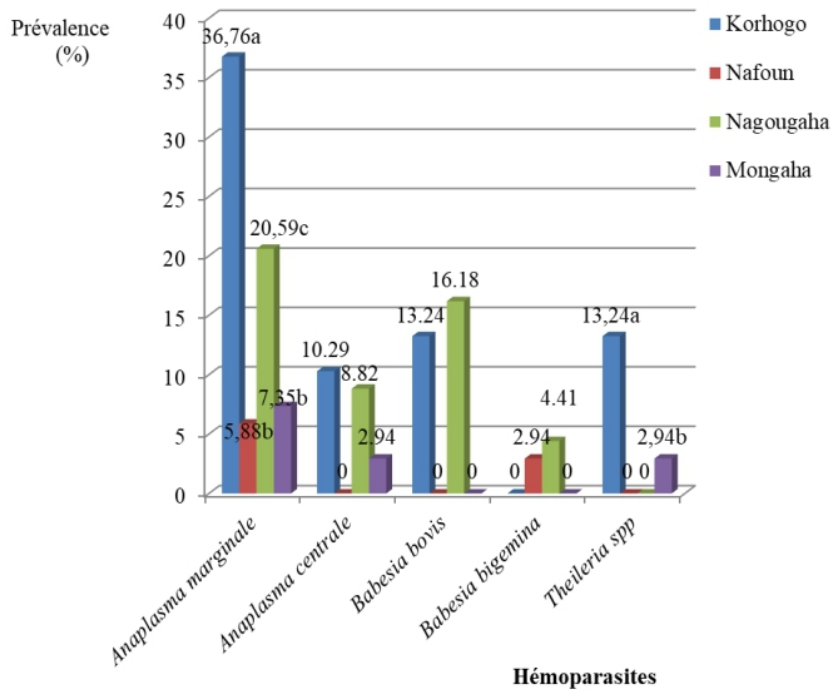

La différence de lettres ( $a, b$ et $c$ ) indiquées, montre qu'il y a une différence significative entre les prévalences des hémoparasites dans les différentes localités.

Figure 7 : Prévalence des hémoparasites chez les bovins en fonction de la localité de prélèvement

Prévalence des hémoparasites chez les ovins en fonction de la localité de prélèvement

La figure 8 montre que Anaplasma marginale infecte les ovins des localités de Nafoun (26,56\%), Korhogo (25\%) et Lokoli (20,31\%) avec une différence statistique non significative $(\mathrm{p}<0,05)$. Cependant, les ovins de la localité de Lokoli sont plus infectés par Anaplasma centrale $(18,75 \%)$ suivis des ovins des localités de Korhogo (4,69\%) et Nafoun (3,13\%). La différence de prévalence d'infection des ovins par ce parasite est significative ( $\mathrm{p}$-value $=$ 0,00037 ) entre les trois localités. Babesia ovis et Babesia bigemina infectent respectivement les ovins des localités de Lokoli $(6,25 \%)$ et Nafoun $(1,56 \%)$ à des prévalences faibles. 


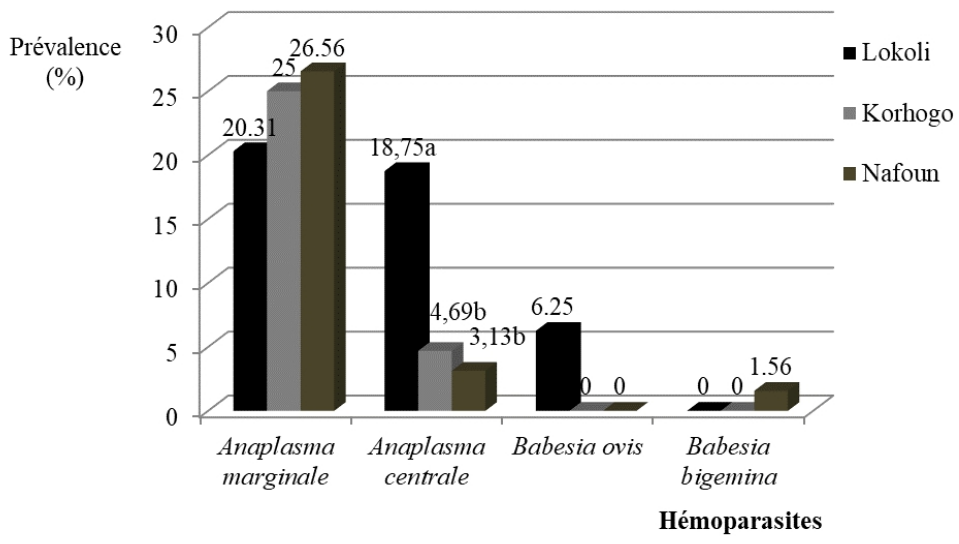

La différence de lettres ( $\mathrm{a}$ et $\mathrm{b}$ ) indiquées, montre qu'il y a une différence significative entre les prévalences des hémoparasites.

Figure 8 : Prévalence des hémoparasites chez les ovins en fonction de la localité de prélèvement

\section{Discussion}

Les bovins et les ovins ont été les ruminants qui ont fait l'objet d'analyse de routine au Laboratoire Régional de Korhogo. La présence de ces espèces animales dans la zone d'étude s'expliquerait par une disponibilité accrue de l'herbe et de la densité en élevage des ruminants domestiques dans cette partie nord de la Côte d'Ivoire. Ces résultats sont similaires à ceux obtenus par Tanguy (2008). Selon cet auteur, les départements de Korhogo et Ferkessédougou représentent près de la moitié de l'effectif du cheptel bovin de Côte d'Ivoire.

Concernant les types d'infections, les bovins ont été infectés par trois genres d'hémoparasites (Anaplasma, Babesia et Theileria) et deux genres (Anaplasma et Babesia) chez les ovins. L'infection des bovins et ovins par ces hémoparasites pourrait s'expliquer par une infestation des animaux par les tiques préalablement infectées par ces parasites. Le non-respect des doses de médicament, des programmes de détiquage et de lutte contre les hémoparasites pourrait être à l'origine de la persistance des tiques et des parasites sanguins chez les animaux. Ces résultats sont similaires à ceux obtenus par Yéo et $a l$. (2017) dans une étude conduite dans les élevages de ruminants domestiques de la Région du Poro. Selon ces auteurs, $85,71 \%$ des éleveurs sédentaires injectent au bétail des doses de médicament faibles dans la lutte contre les hémoparasites et la proportion des éleveurs sédentaires optant pour des doses normales est de 14,29\%. En outre, le parasite sanguin du genre Trypanosoma n'a pas été observé chez les bovins et les ovins. L'absence de cet hémoparasite chez ces animaux serait liée à l'effet accru de l'emploi des insecticides agricoles dans la lutte contre les ravageurs de vergers de mangue et d'anacarde 
fortement cultivés dans la zone d'étude. Ces résultats d'étude sont contraires à ceux obtenus par Yéo et al. (2017). Ces auteurs ont observé le genre Trypanosoma chez les bovins à des prévalences faibles (6,94\%) pour Trypanosoma vivax et 2,50\% (Trypanosoma brucei) dans des élevages au Nord de la Côte d'Ivoire. La différence de résultats observés s'expliquerait par le fait que les éleveurs qui ont sollicité le laboratoire pour des analyses ont auparavant administré de l'oxytétracycline à leurs animaux. En effet, selon Camus et Morel (2000), l'injection de l'oxytétracycline à un animal malade lui permettrait d'obtenir rapidement la guérison.

Concernant les espèces de parasites sanguins, Anaplasma marginale a plus infecté les bovins et les ovins. L'infection élevée des bovins et des ovins par Anaplasma marginale serait liée à l'abondance des tiques vectrices notamment la tique Rhipicephalus (Boophilus) microplus chez ces animaux. Ces résultats concordent à ceux obtenus par Achi et al. (2012) et Madder et al. (2007). En effet, Madder et al. (2007) ont identifié pour la première fois cette tique à Azaguié au sud de la Côte d'Ivoire puis Achi et al. (2012) chez les bovins d'élevage dans le nord de la Côte d'Ivoire. Par ailleurs, Yéo et al. (2017) ont identifié chez des bovins du nord de la Côte d'Ivoire une infection élevée de Anaplasma marginale (76,94\%). Par contre, les résultats de la présente étude diffèrent de ceux obtenus par Farougou et al. (2007). Selon ces auteurs, Babesia bigemina (57\%) infecte plus les bovins d'élevage du Nord de Bénin suivi de Theileria (46,5\%). La différence de résultats obtenus pourrait s'expliquer par le climat qui diffère d'un pays à l'autre.

Parlant du stade physiologique des bovins, les taurillons et les génisses de 1 à 3 ans ainsi que les taureaux et les vaches de plus de 3 ans d'âge sont les plus infectés par Anaplasma marginale. Les veaux et les velles de moins d'un an sont les moins infectés. L'élévation de l'infection chez ces catégories de bovins s'expliquerait par leur plus grande exposition sur le pâturage probablement infesté par les tiques du genre Rhipicephalus (Boophilus) microplus. En outre, la faible infection des veaux pourrait être liée au fait qu'ils sont parqués au parc et subissent moins d'infection par Anaplasma marginale. Par contre, les femelles sont plus infectées que les mâles. Ce fait s'expliquerait par le nombre élevé des femelles dans les élevages. Les résultats obtenus sont similaires à ceux obtenus par Djakaridja et al. (2014) dans une étude conduite dans les élevages des régions des Savanes et de la vallée du Bandama en Côte d'Ivoire. Ces auteurs ont obtenu 78,76\% chez 178 femelles infectées par Anaplasma marginale et 73,13\% chez 98 mâles infectés par cet hémoparasite. Cette tendance est observée chez les ovins. En effet, Anaplasma marginale infecte plus les adultes et les jeunes ovins. Les agneaux et les agnelles sont moins infectés par rapport aux adultes et aux jeunes. Cette infection élevée chez ces catégories d'ovins serait liée à une infestation importante des tiques vectrices infectées. Comme chez les bovins les femelles 
sont plus infectées et cela pourrait se justifier par le nombre élevé des femelles dans les élevages par rapport aux mâles.

Concernant les localités d'étude, les bovins et les chiens de la localité de Korhogo sont plus infectés par Anaplasma marginale tandis que les ovins de la localité de Lokoli sont les plus infectés par Anaplasma centrale. Ces différences d'infection des animaux s'expliqueraient par le fait que ces localités constitueraient un couloir de transhumance des bovins des pays frontaliers (Mali et Burkina Faso) avec pour conséquence l'infestation d'un grand nombre d'animaux d'élevages locaux.

\section{Conclusion}

Cette étude a permis de montrer que les hémoparasites notamment ceux du genre Anaplasma et Babesia infectent aussi bien les bovins que les ovins. Le genre Theileria infecte exclusivement les bovins. Le parasite sanguin du genre Anaplasma infecte plus les animaux. Par ailleurs, Anaplasma marginale infecte le plus les animaux. Les taurillons et les génisses de 1 à 3 ans ainsi que les taureaux et les vaches de plus de 3 ans d'âge sont les plus infectés par Anaplasma marginale. Les veaux et les vêles de moins d'un an sont les moins infectés. En outre, Anaplasma marginale infecte plus les adultes et les jeunes ovins. Les agneaux et les agnelles sont moins infectés par rapport aux adultes et aux jeunes. Comme chez les bovins, les femelles chez les ovins sont plus infectées par cet hémoparasite. Ce parasite est présent dans les cinq localités qui ont fait l'objet d'enquête. En outre, Anaplasma centrale infecte plus les ovins de la localité de Lokoli. L'identification de ces parasites sanguins permettra de mieux choisir les médicaments vétérinaires appropriés afin d'améliorer les productions des ruminants domestiques et réduire les dépenses effectuées par les éleveurs.

\section{Remerciement}

Les remerciements vont à l'endroit des agents vétérinaires privés installés dans les départements et sous-préfectures ainsi que les techniciens supérieurs de recherche du Laboratoire Régional de Korhogo impliqués dans l'obtention de ces résultats.

\section{Conflit D'interet}

Les auteurs ne déclarent aucun conflit d'intérêt.

\section{References:}

1. Achi, Y.L., Koné, P., Stachurski, F., Zinsstag, J., Betschart, B. 2012. Impact des tiques sur des bovins métissés dans le Nord de la Côte d'Ivoire. Bulletin Animal Health Production Africa 60(2), 109-118. 
2. Adamu, B. S. et Balarabe, L. M. 2012. Prevalence of haemoparasites of sheep and goats slaughtered in bauchi abattoir. International Journal of Applied Biological Research, 4(1\&2): 128 - 133.

3. Djakaridja, B., Yao, K. P., Gragnon, B. G., Acapovi-Yao, G. et Mavoungou, J. L. 2014. Situation épidémiologique des hémoparasittes des bovins dans deux zones d'élevage de la Côte d'Ivoire : cas des anciennes régions des savanes et de la vallée Bandama. Revue de Médecine Vétérinaire, 165 : 297 - 303.

4. Farougou, S., Tassou A. W., Tchbodé, D. M., Kpodenou, M., Boko, C et Youssao, A. K. I. 2007. Tiques et hémoparasites du bétail dans le nord-Bénin. Revue de Médecine Vétérinaire, 158 : 463 - 467.

5. Farougou, S., Doko A. S., Sankamaho, I., Codjia, V. 2012. Prevalence of Trypanosome Infections in Cattle and Sheep in the Benin's West Atacora Agro-ecological zone, Tropicultura, 30(3): 141 - 146.

6. Goodman, J.L., Dennis, D.T. et Sonenshine, D.E. 2005. Tick-borne diseases of humans. ASM Press, Washington, DC, USA. 11(11), 440p.

7. Lachance, S. et Balenghien, 2012. Insectes vecteurs de maladies en élevage de ruminants : Le Canada est-il à risque ? Collaboration spéciale Montpellier, France, 29(22), 355p.

8. Madder, M., Thys, E., Geysen, D., Baudoux, C. et Horak, I. 2007. Boophilus microplus ticks found in West Africa. Experimental \& Applied Acarology 43, 233-234 (Short communication).

9. Oguz, E. 2008. Maladie de Lyme : épidémiologie nationale et international, prévention actuelle et perspective. Thèse de la faculté des sciences pharmaceutiques et biologie, Nancy, France, 154p.

10. OIE, 2008. Manuel des tests de diagnostic et des vaccins pour les animaux terrestres. 2.6, éd., Paris, France, 10p.

11. Stachurski, F., Adakal, H. et Desquesnes, M., 2004. Maladie transmise par la tique Amblyomma variegatum. La cowdriose: épidémiologie et contrôle. Santé animale en Afrique de l'Ouest. Fiche n ${ }^{\circ} 11$, CIRAD, Burkina Faso, 8p.

12. Tanguy-le Guen, 2008. "'Le développement agricole et pastorale du nord de la Côte d'Ivoire: Problème de coexistence', les cahiers d'outre-mer, pp : .226-227.

13. Yabile R. K., 2011. Promotion de l'agriculture vivrière et croissance économique de la Côte d'Ivoire: quelle relation ? Agronomie Africaine, $23: 259$ - 272.

14. Yéo, N., Karamoko, Y., Soro, D., Zouh Bi, Z. F. et Traoré, S. I. 2017. Elevages de bétail dans la région du Poro (Côte d'Ivoire) : Caractérisation et modalités de lutte contre les pathogènes transmis par les tiques. International Journal of Biological and Chemical Sciences, 11(1): 237-246. 
15. Yéo, N., Karamoko, Y., Soro, D., Zouh Bi, Z. F., Okon, A. J. L., Gragnon, B. G. 2017. Prevalence of Trypanosoma, Babesia and Anaplasma in cattle reared in the North of Côte d'Ivoire. International Journal of Biosciences, 10(2): 21-28. 